Introduction The world's population is ageing. This creates a need to work for longer, both for income and to provide an adequate labour force. For those employed in predominantly physically demanding jobs this means they are prolonging their exposure to risk factors known to increase the likelihood of a musculoskeletal injury when their work capacity may be declining. The work ability of older workers has been most frequently assessed using the Work Ability Index (WAI). Several studies have reported significant associations between low WAI scores and sickness absence and early retirement. Relatively fewer studies have examined associations between WAI scores and specific workplace risk factors. The purpose of this study was to investigate the association between a range of workplace risk factors and the WAI scores in a cohort of workers employed in physically demanding jobs.

Methods A cross-sectional survey of workers employed in physically demanding roles within a local government council was undertaken. The survey instruments included questions on demographic and employment characteristics, physical and psychosocial risk factors, pain and discomfort, and the Work Ability Index.

Result The survey was completed by $155 / 245$ of eligible workers - a $63 \%$ response rate. Respondents had a mean age of 44 years. They were predominantly male $(86 \%)$ with an average length of employment of 12 years. Bivariate regression analyses were undertaken to examine the relationship between WAI scores and age, pain/discomfort, levels of stress, irritation, job satisfaction, work-life balance, and 49 other work environment risk factors. Significant associations with WAI scores were seen for age, pain/discomfort and physical and psychosocial risk factors. The WAI score was reduced by more than 2.5 points for those who reported higher levels of exposure to a range of physical and psychosocial risk factors compared with those who reported lower levels of exposure.

Discussion It was found that the WAI provided a useful means to identify a range of workplace risk factors which, if addressed, could inform the development of interventions to maintain a healthy, older workforce. It is proposed that tailoring interventions using this approach should enhance their effectiveness.

\section{AGEING AND SHIFTWORK}

${ }^{1}$ Clas-Håkan Nygård*, ${ }^{2}$ Frida Marina Fischer. ${ }^{1}$ Faculty of Social Sciences, Health Sciences, University of Tampere, Tampere, Finland; ${ }^{2}$ School of Public Health, University of São Paulo, São Paulo, Brazil

\subsection{6/oemed-2018-ICOHabstracts.99}

Aim of this special session Ageing and shiftwork is a rather new and 'hot topic' because of the intentions to prolong working lives in many countries. Because shiftwork could be very demanding for older employees it need more attention than earlier.

${ }^{1}$ Anne-Helene Garde, ${ }^{2}$ Janet Barnes- Farrell, ${ }^{3}$ Jeanne Duffy, ${ }^{4}$ Karin Proper, ${ }^{5}$ Jill Dorrian, ${ }^{6}$ Prakash KC

${ }^{1}$ The National Research Centre for the Working Environment, Copenhagen, Denmark

${ }^{2}$ University of Connecticut, Storrs, CT, USA

${ }^{3}$ Division of Sleep and Circadian Disorders, Brigham and Women's Hospital, Boston, MA, USA

${ }^{4}$ National Institute for Public Health and the Environment, Bilthoven, The Netherlands

${ }^{5}$ University of South Australia, Adelaide, Australia
${ }^{6}$ Faculty of Social Sciences (Health Science), University of Tampere, Tampere, Finland

\section{7 a PERMANENT NIGHT WORK, AGE AND SICKNESS ABSENCE}

${ }^{1,2} \mathrm{AH}$ Garde, ${ }^{1,2} \mathrm{~A} M$ Hansen, ${ }^{3} \mathrm{HA}$ Kolstad, ${ }^{4} \mathrm{~J}$ Hansen, ${ }^{1} \mathrm{HB}$ Nielsen, ${ }^{1} \mathrm{AD}$ Larsen. ${ }^{1}$ The National Research Centre for the Working Environment, Copenhagen, Denmark; ${ }^{2}$ Department of Public Health, Copenhagen University, Copenhagen, Denmark; ${ }^{3}$ Danish Ramazini Centre, Department of Occupational Medicine, Aarhus University Hospital; ${ }^{4}$ The Danish Cancer Society, DK-2100 Copenhagen, Denmark

\subsection{6/oemed-2018-ICOHabstracts. 100}

Introduction Night shift work is associated with adverse health effects. Yet, some persons prefer working permanent night shifts and it is speculated that they tolerate night work better than others. The aim of is to study associations between permanent night work, age and sickness absence. Due to selfselection out of night work over time by those who experience negative effects of night work, we hypothesised that older workers with permanent night work are 'healthy workers' with less sickness absence compared to other groups.

Methods Information on working hours, age (20-34 years, 35-49 years and >50 years) and sickness absence was obtained from the Danish Working Hour Database, which contains daily information on starting and ending time of working hours based on payroll data for all employees at Danish public hospitals (2008-2015). For each year with $>50$ workdays, individual schedules were classified as permanent day, evening or night ( $>88 \%$ of work days with night work), 2-shift (day/evening, day/night or evening/day) or 3-shift (day/ evening/night). We applied linear regression with individual as random intercept (participants served as their own controls) for employees $(n=5774)$ with at least one year of permanent night work.

Results Employees aged >50 years had 25.6 sickness days/year when working permanent night, which is more than in all other schedules. In comparison, employees had $9.0 \quad(\mathrm{sd}=1.0)$ fewer days when working permanent day, $7.3 \quad(\mathrm{sd}=2.4)$ for permanent evening, $6.3(\mathrm{sd}=0.9)$ for day/evening, 3.2 ( $\mathrm{sd}=0.6)$ for day/night, $5.3(\mathrm{sd}=1.0)$ for evening/night, and $5.5(\mathrm{sd}=0.7)$ for day/evening/night. There was no interaction effect between schedule and age.

Discussion Employees had more sickness absence when working permanent night work compared to any other schedule. The association was not modified by age and did therefore not indicate that older employees with permanent night work tolerate night work better than others.

\section{7b PSYCHOSOCIAL AND HEALTH IMPLICATIONS OF AROUND THE CLOCK OPERATIONS FOR CORRECTIONS OFFICERS}

${ }^{1} \mathrm{JL}$ Barnes-Farrell, ${ }^{1} \mathrm{D}$ Tubbs, ${ }^{2} \mathrm{~A}$ Dugan, ${ }^{2} \mathrm{~S}$ Namazi, ${ }^{2} \mathrm{M}$ Cherniack. ${ }^{1}$ University of Connecticut, Storrs, CT, USA; ${ }^{2}$ University of Connecticut Health Centre, Farmington, CT, USA

10.1136/oemed-2018-ICOHabstracts.101

Introduction Corrections officers in state and federal prisons are faced with physical and psychosocial work demands that create challenges for maintaining high levels of workability, health and personal well-being. Furthermore, features of 